Cinémas

Revue d'études cinématographiques

Journal of Film Studies

\title{
Narration et temporalité dans Moderato cantabile
}

\section{André Gardies}

Volume 4, numéro 1, automne 1993

\section{Écrit/Écran}

URI : https://id.erudit.org/iderudit/1000113ar

DOI : https://doi.org/10.7202/1000113ar

Aller au sommaire du numéro

\section{Éditeur(s)}

Cinémas

\section{ISSN}

1181-6945 (imprimé)

1705-6500 (numérique)

Découvrir la revue

Citer cet article

Gardies, A. (1993). Narration et temporalité dans Moderato cantabile. Cinémas, 4(1), 88-102. https://doi.org/10.7202/1000113ar

\section{Résumé de l'article}

Raconter, à l'écrit comme à l'écran, c'est négocier un temps, celui des événements de l'histoire, dans un autre, celui propre à l'actif narratif. Cependant, du roman au film, en raison particulièrement des différences de matière de l'expression, les stratégies mobilisées affichent de notables particularités Moderato cantabile, le roman de Marguerite Duras et le film de Peter Brook, en fournissent un bon terrain d'analyse. L'image peut-elle reprendre à son propre compte le travail tout à fait singulier du livre de Duras sur la temporalité? C'est en opérant sur la durée, lieu où se manifeste pleinement la spécificité du cinématographique, qu'il pourra avancer ses propres réponses. 


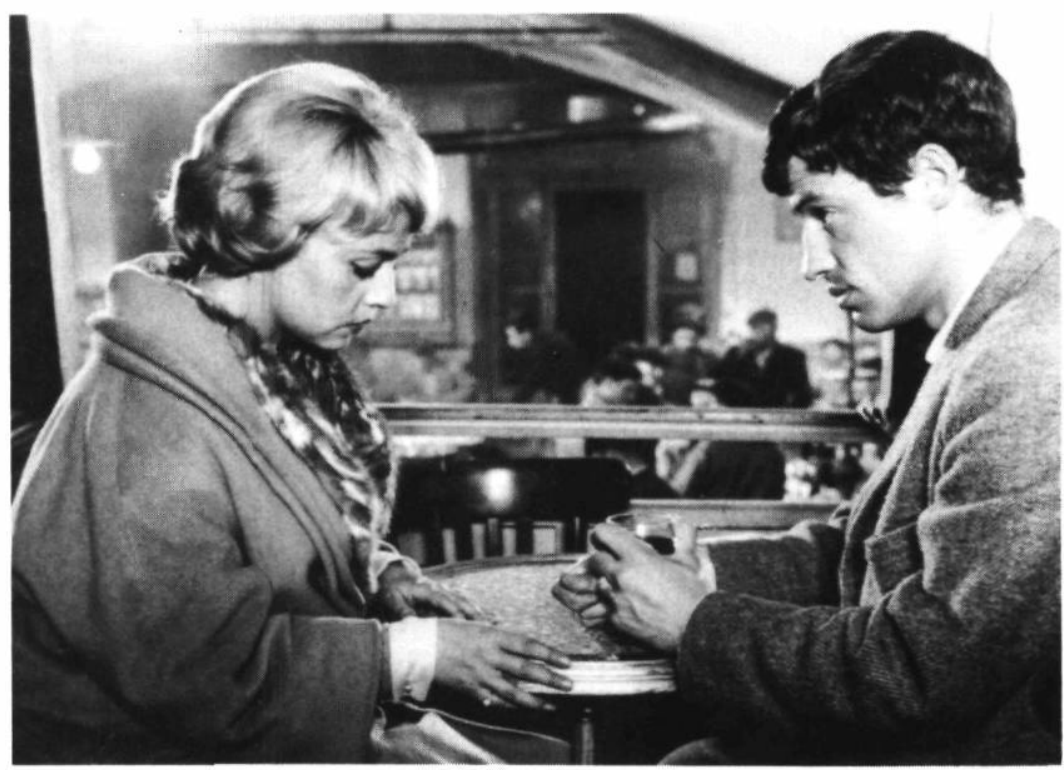

Moderato cantabile de Peter Brook Coll. Cinémathèque québécoise 


\section{Narration et temporalité dans Moderato cantabile}

\section{André Gardies}

\section{RÉSUMÉ}

Raconter, à l'écrit comme à l'écran, c'est négocier un temps, celui des événements de l'histoire, dans un autre, celui propre à l'actif narratif. Cependant, du roman au film, en raison particulièrement des différences de matière de l'expression, les stratégies mobilisées affichent de notables particularités. Moderato cantabile, le roman de Marguerite Duras et le film de Peter Brook, en fournissent un bon terrain d'analyse. L'image peutelle reprendre à son propre compte le travail tout à fait singulier du livre de Duras sur la temporalité? C'est en opérant sur la durée, lieu où se manifeste pleinement la spécificité du cinématographique, qu'il pourra avancer ses propres réponses.

\section{ABSTRACT}

Telling a story, in script or on screen, means negotiating a passage from one time frame, that of the events recounted in the story, into another, that of the narrative act. However between novel and film, because of differences in expressive matter, the strategies employed exhibit notable discrepancies. Moderato cantabile, Marguerite Duras' novel and Peter Brooks' film, offers a promising site for the examination of these differences. Can the image alone perform the same remarkable work performs on temporality as Duras' book? It is by exploiting duration, where cinematographic specificity is fully apparent, that it manages to pose its own solutions. 
Pour qui lit attentivement Moderato cantabile, le roman de Marguerite Duras, un trait singulier ne devrait pas manquer de le frapper : deux temps diégétiques s'y côtoient, dans une relation souvent concurrentielle.

Le premier, c'est celui de la rencontre d'Anne Desbaresdes et de Chauvin, ces dix jours au cours desquels se vit dans le quotidien un amour hors du quotidien. Le second, c'est celui de la passion prêtée au couple inconnu, à la fois suspendue et prolongée indéfiniment par la mort. Deux couples, deux amours, deux récits : rien de surprenant, dira-t-on, à ce que se développent deux temporalités.

C'est que la singularité est ailleurs : dans l'opposition entre un temps précis, daté, presque minuté (le premier) et un temps, le second, sans limites, sans repères, sans cesse en transformation, comme s'il était en continuelle expansion. Opposition entre un temps chronique ${ }^{1}$ - le temps socialement marqué, celui des horloges et des calendriers - et un temps subjectif, directement en prise sur l'imaginaire des deux protagonistes. Opposition entre un temps qui se calcule, qui se mesure, qui se gère et un temps hors temps, celui de la passion absolue.

Ainsi la voix narratrice, qui prend en charge l'essentiel du récit premier, multiplie les précisions tout en soulignant le déroulement linéaire et comme irréversible de ces dix jours de la «liaison» entre Anne et Chauvin :
«(...) au cœur de l'après-midi de ce printemps» (p. 8); «(...) le crépuscule commença à balayer la mer» (p. 11); «Le lendemain... à l'heure déjà passée où chaque vendredi ils allaient dans ce quartier» (p. 17); "C'était un samedi» (p. 21); «Six heures déjà, annonça la patronne» (p. 22); «Le tremblement était encore plus fort que trois jours auparavant» (p. 28); "Le lendemain encore» (p. 38); etc.

Moyennant un minimum d'attention, le lecteur peut dater et mesurer chacun des épisodes quotidiens d'Anne Desbaresdes. Sa rencontre avec Chauvin a lieu un samedi; leur histoire s'achève un lundi, dix jours plus tard. En outre, l'ordre des chapitres suit le déroulement chronologique avec une relative régularité; à l'exception notable, toutefois, du second vendredi qui occupe à lui seul trois chapitres.

Et pourtant l'effet général que produit la lecture du roman est nettement celui d'une grand fluidité, flottant, indécidable, sans véritable début ni véritable fin, où passé, présent et avenir se mêlent, se chevauchent, se substituent l'un à l'autre; un temps dans lequel se fond le lecteur. 
C'est que la temporalité du second récit contamine celle du premier, la brouille, la submerge, l'emporte dans sa croissante expansion. Car elle est, elle, non plus celle des gestes, des actes et des habitudes du quotidien, mais celle de l'imaginaire passionnel. Pourtant cela commence simplement, à la façon d'une enquête qui serait avivée par la curiosité :

«C'était un crime, dit l'homme... - Je vois... Je me le demandais voyez-vous...» (p. 18); «Ce cri était si fort que vraiment il est bien naturel que l'on cherche à savoir... - Ce que je sais c'est qu'il lui a tiré une balle dans le cœur» (p. 19).

Le meurtre a eu lieu la veille et les premiers propos qu'échangent Anne et Chauvin sont donc naturellement tournés vers ce passé récent. Mais très vite on remonte dans le temps :

«lls s'aimaient, dit-il... Lui travaillait à l'arsenal. Elle, je ne sais pas...» (p. 20)

pour quitter bientôt l'énoncé des faits avérés et s'ouvrir au mode hypothétique :

«- Peut-être avaient-ils des difficultés, ce qu'on appelle des difficultés de cœur alors?» (p. 20); «Mais peut-être n'est-ce pas en raison de ces difficultés qu'il l'a tuée, qui sait?» (p. 21).

À partir de ce moment-là, le champ est ouvert : puisque chacun en sait si peu sur ce crime, sur cette histoire, elle est donc «libre»; Anne et Chauvin peuvent se l'approprier, la faire leur. Ils vont alors l'inventer, lui donner une origine, une durée, un futur hypothétique :

«lls s'étaient connus par hasard dans un café... Et ils ont commencé à se parler de chose et d'autre» (p. 30); «Je crois qu'ils ont passé beaucoup de temps ensemble pour en arriver là où ils étaient» (p. 33); "Ils ne savaient pas, avant d'y aller, qu'ils en viendraient là si vite. Qu'au bout de quelques jours, il serait obligé de la chasser si souvent » (p. 63).

Bientôt, par une sorte d'osmose, elle va gagner leur propre histoire, ou plus exactement ce qui se passe entre Anne et Chauvin, pour être dit et donc exister, va se couler dans ce moule resté creux et disponible. Les similitudes d'abord sont esquissées :

«Une fois, il me semble bien, oui, une fois j'ai dû crier un peu de cette façon... dans un café, peut-être même dans ce café-ci qu'ils fréquentaient tous les deux» (p. 30); «Une certaine nuit, ils tournent et retournent dans leur chambre, ils deviennent comme des bêtes enfermées, ils ne savent pas ce qui leur arrive» (p. 40). 
puis, le processus d'identification s'accentuant, une véritable fusion s'opère où le «elle» désigne à la fois l'inconnue morte et, bien sûr, Anne Desbaresdes :

«- C'est là, dans cette maison, qu'elle a appris ce que vous disiez qu'elle était, peut-être par exemple...

— Oui, une chienne, l'arrêta encore Chauvin» (p. 64).

L'histoire du couple inconnu devient, bien entendu, celle d'Anne et de Chauvin, de ce couple qui ne se connait pas, mais qui se révèle, puis existe en s'inventant. Dès lors, le temps du récit premier, celui du quotidien, des rencontres renouvelées durant dix jours, en dépit de la précion d'horloge, va être habité par un temps fabuleux, un temps libre des contingences, un temps qui se nourrit de chaque événement vécu pour devenir un temps sans limites où passé, présent et futur s'annulent dans un temps absolu, celui de la passion incandescente. Alors, lorsqu'à la page 84 prend fin le roman et qu'au dixième jour se défait le couple, le lecteur de Moderato cantabile a perdu ses repères temporels; il a éprouvé progressivement le sentiment d'une durée indéfinie à laquelle seule la mort, origine de l'histoire et destin inéluctable, peut apporter un terme.

Pour qui, maintenant, regarde et écoute Moderato cantabile, le film de Peter Brook, il ne peut manquer d'être frappé par deux traits singuliers. D'une part la double temporalité du roman y est ramenée, pour l'essentiel, à l'axe temporel propre aux rencontres quotidiennes d'Anne et de Chauvin. D'autre part, ce qui reste encore du double récit s'articule sur une opposition entre l'image et le verbal. Revient alors à la bande-images le soin de produire un effet de durée indéfinie tout en marquant la succession des rencontres.

Cela représente une telle transformation que l'on ne peut manquer de s'interroger sur les raisons de ce choix, avant d'en examiner ensuite les conséquences.

Pour autant, il ne s'agit pas d'aller à la recherche des intentions du réalisateur ni de convoquer quelque autorité de l'auteur; cette démarche ne concerne pas le sémionarratologue. L'examen des conditions de transcription intersémiotique (ce qu'est, à un certain niveau, toute adaptation cinématographique) délimitera le champ des interrogations.

L'une des différences majeures qui distingue le roman du film, la chose est bien connue, tient à la matière de l'expression : homogène dans un cas (la langue écrite), hétérogène dans l'autre (composée d'images mouvantes, de mentions écrites, de verbal, de bruitage et de musique). À elle seule, cette différence crée un 
réseau de contraintes si prégnantes qu'elles expliquent, déjà, nombre des transformations repérables dans le film de Peter Brook.

En ce sens, les premières lignes de Moderato cantabile sont exemplaires:

- Veux-tu lire ce qu'il y a d'écrit au-dessus de ta partition? demanda la dame.

- Moderato cantabile, dit l'enfant.

La dame ponctua cette réponse d'un coup de crayon sur le clavier.

Deux «voix» se font entendre : celle des personnages, celle du narrateur. Et le flux linéaire de l'écrit leur impose un rapport d'exclusion : quand l'une parle, l'autre doit s'absenter. Leur mode de présence au texte ne peut être que celui de l'alternance (alors qu'au cinéma, on le sait, je puis entendre ce que disent les personnages «en même temps» que je vois ce qu'ils font). Précisément le roman de Marguerite Duras joue totalement de cette caractéristique du médium : quand les personnages dialoguent, leurs propos, tout en étant matériellement homogènes de la parole du narrateur (c'est de la langue écrite dans les deux cas), sont toujours émis dans une situation diégétique singulière (au café, chez Mlle Giraud, au cours des promenades, etc.) rapportée, elle, par le narrateur et constituant le récit premier. Or le récit du couple inconnu et de sa passion mortelle ne sera rapporté-inventé que par Anne et Chauvin, à travers leurs dialogues. Il se trouve donc en situation d'emboîtement par rapport au récit premier. En outre, et c'est là un trait propre à ce roman, ces dialogues sont quantitativement plus importants que la part accordée à la voix narratrice, car l'action de Moderato cantabile, pour la résumer cavalièrement, consiste en ce que deux êtres se rencontrent, parlent et vivent par leur parole. Si l'on ajoute à cela qu'en même temps qu'ils inventent l'histoire du couple inconnu, c'est leur propre histoire qu'ils font exister, grâce à l'ambivalence constitutive du langage, on mesurera les difficultés que comportait cette adaptation cinématographique.

Le risque premier était celui du théâtre filmé. En effet on sait que l'image mouvante est sémiologiquement première au cinéma (sans elle point de film). C'est elle, ici, qui prend en charge le récit premier : elle met en situation Anne Desbaresdes et son fils, sa rencontre avec Chauvin et leurs échanges verbaux. L'action qu'elle est alors susceptible de montrer, de mettre en scène, est seulement celle d'un dialogue. Cependant, une autre solution cinématographique, fort courante au demeurant, reste possible : visualiser le contenu de leurs propos, suivant le principe de 
l'emboîtement des récit. L'histoire du couple inconnu pourrait donc être non pas racontée verbalement, mais filmée et donc mise en images.

Nul besoin d'insister sur le contresens que représenterait une telle option : en "matérialisant» le couple inconnu, en le faisant exister de manière autonome, l'ambivalence sur laquelle travaille le roman serait littéralement court-circuitée; la relation d'osmose, de mimétisme et d'identification par laquelle se constitue le couple Anne et Chauvin s'en trouverait évacuée. Le «moule» de l'histoire des deux inconnus étant «plein», Anne et Chauvin ne pourraient plus se glisser à l'intérieur.

Éviter le piège du théâtre filmé d'un côté, de l'autre refuser le contresens de la visualisation, la marge d'action est étroite. La solution retenue par le film consiste, d'une part, à ne faire exister l'histoire du couple inconnu que par l'échange verbal et, d'autre part, à renvoyer sur le récit premier une large part des effets que produisait, dans le roman, le récit second. Pareil choix entraîne de multiples conséquences.

$\mathrm{Au}$ niveau scénaristique d'abord. Alors que dans le roman toutes les rencontres d'Anne et Chauvin se déroulent dans le café, théâtre du meurtre, le film, lui, multiplie les lieux : le long des quais, dans la maison abandonnée au-delà de l'estuaire, sur le mail, sur la rive du fleuve, sous la grande allée de platanes. Et cela ne répond pas seulement à quelque souci anecdotique de variété. Il s'agit de rendre sensible (visuellement et auditivement) l'évolution continue de la relation entre Anne et Chauvin. Dans le roman, cette même évolution s'inscrit dans les propos qu'échangent les personnages, à travers justement l'histoire qu'ils inventent, le travail souterrain d'identification qu'elle engendre et l'ambivalence même du langage. Dans le film, la succession et l'ordre des lieux seront l'un des moyens susceptibles de matérialiser cette évolution, notamment par les variations de tonalité propre à chacun d'eux : lieux diurnes, crépusculaires ou nocturnes, vacuité d'une maison abandonnée, intimité précaire d'un banc public, fluidité étale du fleuve, protection illusoire des grands platanes de l'allée, etc. La fragilité de leur passion s'en trouve soulignée jusqu'à la vanité, non sans quelque dimension tragique.

Cependant, c'est probablement dans le traitement de la temporalité que se lisent les transformations les plus remarquables.

Par référence aux travaux de Gérard Genette, celle-ci peut être envisagée sous trois aspects : la durée, I'ordre et la fréquence. Le premier surtout retiendra notre attention, car il 
pose, au-delà de la singularité propre à l'adaptation de Moderato cantabile, un certain nombre de questions concernant la narratologie et plus précisément la narratologie filmique.

Toutefois un regard, même rapide, sur la façon dont le film résout les problèmes d'ordre et de fréquence ne sera pas dénué d'intérêt. En transférant, pour l'essentiel, sur l'axe temporel du seul récit-images, la double temporalité qui structure le roman, le film se trouve confronté à quelques difficultés auxquelles, du reste, il apporte des réponses non dénuées d'originalité.

Ainsi en va-t-il pour l'ordre. Dans l'ensemble, il épouse l'ordre chronologique, celui du récit premier dans le texte de Marguerite Duras. Il introduit cependant, de façon très ponctuelle il est vrai, quelques distorsions qui viennent corriger, en la densifiant, la continuité linéaire. Ainsi, vers la fin du premier tiers du film, tout de suite après la rencontre dans la maison abandonnée, un long travelling fait défiler les grilles extérieures d'une belle demeure. Il fait nuit; on devine une fenêtre éclairée; et tandis que la caméra progresse, on entend un faible crissement de pas. S'agit-il d'une sorte de plan de coupe? Une nouvelle séquence s'ouvre-t-elle? Deux plans brefs suivent : à nouveau une fenêtre éclairée, le parc dans l'obscurité. Puis, en plan large, on devine au loin et dans la nuit, Chauvin assis sur un banc, en bordure de l'estuaire. Passage assez bref, quelque peu énigmatique, de surcroît difficile à localiser dans le temps des événements. Il apparaît comme une sorte d'enclave indécidable. Deux épisodes plus tard (après la reconstitution du crime et l'enfant jouant solitaire dans le parc), Chauvin, qui a retrouvé Anne, lui dit : «Je suis passé devant chez vous cette nuit.» Un effet d'analepse interne se produit aussitôt : le lent travelling sur la grille, le crissement des pas perdent maintenant leur caractère énigmatique. C'était le point de vue objectif de Chauvin au moment de sa promenade nocturne. En fait l'ordre chronologique de la bande-images était respecté, seule était suspendue son interprétation. Les propos de Chauvin invitent à un retour en arrière d'assez grande ampleur textuelle et produisent chez le spectateur le sentiment d'une plus grande densité.

Une autre forme d'anachronie intervient encore, plus loin, lors de la réception donnée chez les Desbaresdes. Devant le comportement étrange (étrange eu égard aux conventions sociales) d'Anne, l'une des invitées émet l'hypothèse que la cause en pourrait être la fleur de magnolia qu'elle porte accrochée à sa robe. La présence effective de cette fleur appelle (plus précisément rappelle) ce moment antérieur où Chauvin parlait à 
Anne de la fleur de magnolia qu'elle avait entre les seins lors de la réception annuelle donnée au personnel de la compagnie. C'est ici un moment antérieur qui fait retour dans le présent de la soirée.

Ces deux exemples (il en est quelques autres dans le film) font apparaître un traitement assez particulier de la chronologie. Il ne s'agit pas d'anachronie au sens que lui donne Gérard Genette, où un événement est raconté maintenant alors qu'il aurait dû l'être avant (c'est l'analepse) ou qu'il devrait l'être plus tard (c'est la prolepse). L'ordre des événements n'est pas bousculé mais le spectateur est invité à établir des rapprochements entre deux moments effectivement séparés; ce faisant, il éprouve l'épaisseur temporelle du texte.

$\mathrm{La}$ fréquence, elle, reçoit une réponse fondée sur le compromis. En effet, tandis que le roman oppose nettement le singulatif (dans le récit premier, où chaque événement est raconté une fois) au régime fréquentatif (dans le récit sur le couple inconnu, où des séries d'événements sont condensées en un seul énoncé ou bien font l'objet de reprises multiples), le film opte pour l'effacement des repères temporels et de la datation. Les événements mis en images (par opposition à ceux racontésinventés par Anne et Chauvin) semblent être traités sur le mode singulatif; cependant, en raison de leur indétermination, ils acquièrent parfois une valeur itérative. C'est le cas, par exemple, des promenades d'Anne avec son fils. Chacune de leur représentation vaut-elle pour un moment unique, toujours singulier, ou figure-t-elle la vie quotidienne, ordinaire, habituelle (donc répétitive) de la mère oisive et de son enfant? Le film maintient cette indécision, d'autant plus qu'elle va ajouter aux effets de durée, qui représentent, eux, le véritable enjeu de cette adaptation.

À partir d'un rapport quantifiable entre le temps du récit et celui de l'histoire s'évalue la durée, selon Gérard Genette. Ce qui est à la fois facile et impossible avec le roman de Marguerite Duras. Je l'ai déjà indiqué, le récit d'Anne et de Chauvin comporte de si nombreuses et insistantes précisions que l'évaluation de la durée ne fait guère difficulté. Le premier chapitre, par exemple, relate en dix pages environ deux heures d'histoire (une heure pour la leçon de piano, une heure approximativement pour le «spectacle» de l'arrestation et le retour jusqu'à la maison). Entre parenthèses, précisons que le film relate ces mêmes événements en 9' 45"; ce qui équivaut, dans les deux cas, au dixième du temps total du récit (10 pages sur 78; 9'45" sur 90'). Les huit chapitres de l'ouvrage 
correspondent à dix jourṣ d'histoire présente (il conviendrait pour être plus précis de tenir compte de l'analepse externe d'un an, lorsque Chauvin évoque sa première «vision» d'Anne). Les ellipses sont de faible amplitude, 48 heures au plus. Analyser la durée de la «liaison» entre Anne et Chauvin ne ferait donc guère difficulté. En revanche, l'autre histoire, celle du couple inconnu, est presque impossible à évaluer; elle n'a pas de durée précise; rapportée-inventée le plus souvent sur le mode hypothétique, elle ne s'embarrasse pas de dates; elle est sans ancrage temporel; simplement elle a eu lieu avant et, par ses conséquences, elle se poursuit encore. Au reste, peut-on même être assuré qu'il s'agit de l'histoire d'un couple inconnu? N'est-ce pas très vite l'histoire d'Anne et Chauvin? Dès lors la durée indéterminée de l'un finit par contaminer, jusqu'à la rendre jusqu'à un certain point inopérante, la précision de l'autre.

Le film, lui, choisit une autre stratégie pour rendre ce sentiment d'une durée indéfinie, en dépit de la brièveté de la rencontre entre Anne et Chauvin. Tout d'abord, comme je viens de l'indiquer, il gomme largement les repères de datation (quelques indications demeurent cependant, comme ce : «déjà sept jours» prononcé dans les derniers moments du film par Chauvin). S'il est encore relativement aisé au début, l'établissement du rapport entre le temps du récit et celui de l'histoire deviendra de plus en plus problématique à mesure qu'avance le film car diverses procédures, au niveau de la bandeimages, seront déployées afin de produire indétermination et durée temporelles.

Toutefois, avant de les analyser, il importe de rappeler à nouveau quelques caractéristiques du médium cinématographique. Envisagé sous l'aspect de la durée, le temps filmique (celui du récit et non celui de l'histoire) offre une singularité remarquable : il procède paradoxalement de l'intermittence, du clignotement, et cela doublement. D'abord, au sein du plan, dans le passage de photogramme à photogramme. On le sait, le mouvement lisible à l'image n'est qu'un mouvement apparent ayant pour origine la projection, à cadence standardisée, de 24 images fixes par seconde. Si le phénomène a été maintes et maintes fois analysé sous l'angle du mouvement, ses conséquences au plan de la temporalité ont été moins souvent considérées. La perception de la durée, celle du déplacement d'un objet sur l'écran par exemple, correspond en fait à la perception d'une continuité. En ce sens, l'unité de durée est une unité de continuité. Et celle-ci est comprise entre deux discontinuités. Or, parce que la discontinuité réelle inscrite entre 
chaque photogramme qui le compose est rendue imperceptible, tout plan est perçu comme continu et donc affecté d'une durée. En réalité il est constitutivement intermittent.

Au niveau de l'unité discursive supérieure, celle du syntagme (au sens de la sémiologie metzienne), on retrouve un principe semblable. Même si le segment est perçu «narrativement» comme continu (c'est le cas de la scène, de la séquence ordinaire aussi bien que par épisodes, ou encore du syntagme alterné), il est constitué de discontinuités, celles des raccords entre les plans qui, toutefois, à la différence des photogrammes, sont perceptibles, même si, dans les conditions ordinaires, ils ne «doivent» pas être vus. La durée filmique, au niveau du récit, du signifiant, est donc singulière : elle prend constamment appui sur la discontinuité, imperceptible dans un cas (celui des photogrammes), perceptible dans l'autre (celui du syntagme).

Mais, dira-t-on, si l'on admet que la perception de la durée correspond d'abord à la perception d'une continuité, comment cela est-il compatible avec la présence d'une discontinuité perceptible? C'est qu'en réalité, au sein du syntagme, la discontinuité n'est que partielle. Raccorder deux plans au sein d'un segment autonome consiste à travailler simultanément les éléments de continuité et ceux marquant la rupture, et l'attention portée au flux narratif et sémantique l'emporte sur la perception du raccord.

Dès lors, la durée travaille à deux niveaux : celui du plan, celui du syntagme. Avec le premier, le temps du récit est (sauf trucage) égal à celui de l'histoire; au niveau du second, des écarts sont possibles. Car le syntagme (la séquence ordinaire par exemple) est de l'ordre du clignotement : il est fait de pleins (chaque plan) et d'ellipses (entre chaque plan). Narratologiquement, ces deux niveaux n'ont pas le même statut. Dans la stricte perspective de la temporalité, l'un ne possède, pour me référer métaphoriquement à la linguistique, qu'une «articulation» de premier niveau (au sein du plan, parce que produite par l'appareillage technologique d'enregistrement et de projection, la durée de l'action coïncide avec la représentation visuelle de cette même action), tandis que le second possède une «double articulation» : la continuité du plan et l'intervalle du raccord entre deux plans. Or cet intervalle n'a pas de valeur propre; il peut donc se charger de n'importe quelle valeur et travailler ainsi, en rapport avec celle propre au plan, la durée du syntagme.

Or le film de Peter Brook use faiblement de cette possibilité. La plupart des épisodes narratifs (les diverses rencontres, les 
promenades ou encore la réception) sont traités sur le mode de la scène (ce type de syntagme où temps du récit et temps de l'histoire sont égaux) ou, pour être plus précis, sur le mode du régime scénique. Et j'entends par là désigner moins l'exacte correspondance entre récit et histoire (toujours un peu illusoire au cinéma) qu'une tendance vers l'équivalence sans qu'elle soit nécessairement atteinte. Par là, le film produit déjà une sorte de durée étale, sans changements de rythme notables, hormis les grandes ellipses qui séparent chacun des épisodes. À vrai dire, pareil choix n'offre rien de fort original; il relève d'un mode narratif assez commun.

Plus intéressant apparaît le traitement interne au plan, dans la mesure où la durée y est produite à partir de contraintes spécifiquement filmiques dont, par conséquent, le seul modèle genettien ne peut rendre compte.

Deux caractéristiques retiennent l'attention. D'une part, la présence, à intervalles plus ou moins réguliers, de plans en mouvement, vides de personnages (le travelling sur la grille déjà mentionné, mais aussi deux autres travellings à la surface du fleuve ou encore sur la balustrade du mail et les arbres de la forêt de pins); d'autre part (et ils sont les plus fréquents), des plans assez longs, comportant peu d'actions et relativement vides. Ces deux types répondent à des stratégies narratives différentes.

Les premiers, grâce à la conjonction de leur mouvement régulier et lent, de leur absence de personnages ainsi que de leur emplacement dans la chaîne filmique, sont là pour dire : il y a du temps qui passe. Ils sont une sorte de représentation pure de la durée indéfinie. Plus précisément, non pas la représentation mais la manifestation : il y a, tandis que je regarde ces images, réellement du temps qui passe. Le mouvement d'appareil, régulier et continu, avec la continuelle transformation de l'image qu'il engendre, est un événement qui a lieu maintenant, au moment où je le regarde, et dont j'éprouve la durée. Ces plans mettent en évidence la dimension phénoménologique propre à la durée cinématographique : l'expérience que je fais ici et maintenant du temps en acte. Expérience qui reçoit aussitôt un sens narratif. Dans ce film-ci, ces plans disent : entre deux moments de l'histoire un certain temps s'est écoulé. Ils signifient donc, mais sur la base d'une expérience phénoménologique grâce à laquelle j'éprouve réellement la durée.

Les seconds, les plus nombreux, répondent à un choix esthétique : le récit premier (celui du couple Anne et Chauvin) est délibérément traité en plans longs qu'animent peu d'actions, sur un décor généralement dépouillé. Sur ces données de base 
s'élabore l'effet de durée. Durée dont il convient, du reste, de préciser le sens. Elle n'est pas, narratologiquement, de même nature que celle qu'étudie Genette. Celle-ci est une durée à "double articulation" puisqu'elle prend en compte le travail des ellipses, alors qu'au niveau où je me situe, celui du plan, seule l'articulation de premier niveau intervient (coïncidence entre le temps de l'action et celui de sa représentation). C'est la durée propre à la monstration, et elle ne se réduit pas à la seule durée chronométrique du plan. On sait qu'un plan d'une minute peut paraître moins long qu'un plan de dix secondes. C'est là un constat banal qui ne manque cependant pas de poser quelque problème sémio-narratologique. Quels sont les traits pertinents susceptibles de produire cet effet de durée?

Le film de Peter Brook, de ce point de vue, est instructif. Ainsi, à titre d'exemple, les deux plans suivants : a) Anne et Chauvin, après la traversée de l'estuaire, s'éloignent de la berge le long d'une route droite; b) dans la maison abandonnée, au premier plan, Anne, immobile, est là, songeuse au milieu d'une pièce vide; au fond, dans une autre pièce, Chauvin apparaît dans l'embrasure d'une porte, puis s'approche d'Anne. Dans ces deux plans s'impose un fort sentiment de durée, mais pour des raisons différentes.

Le premier propose une action itérative (la marche) au sein d'un décor relativement nu, marqué par l'horizontalité (du fleuve, de la berge, de la route). Le second propose une action singulative (Chauvin rejoint Anne) en jouant sur la profondeur de champ : c'est du fond de la maison vide que vient Chauvin. Deux traits pertinents sont ici à l'œuvre : le taux d'information narrative, l'encombrement de l'image.

Dans le premier cas, la marche des personnages s'apparente à une fonction catalyse, au sens de Roland Barthes : elle prépare la fonction cardinale de l'image (ce moment où le récit progresse) entre Anne et Chauvin. Elle comble le temps qui sépare deux noyaux actionnels. De plus, sa dimension itérative (marcher se répète et se déroule tout au long du plan, sans introduire de transformation repérable) ne lui assigne guère de tension particulière : elle est continuité pure du déplacement. On comprend alors que l'effet de durée soit si sensible : du temps passe, sans produire d'information narrative de quelque importance.

En ce sens on pourrait avancer une première règle : au sein du plan (moyennant quelques conditions complémentaires, ceci est peut être extensible au segment, voire au film lui-même), l'effet de durée est inversement proportionnel au taux d'information. 
Plus précisément, et d'une autre manière : plus, dans un temps chronométrique donné, le plan dispense d'informations, moins sensible est l'effet de durée.

Reste une difficulté, et non des moindres : comment évaluer ce taux d'information? Tout laisse à penser néanmoins que la piste ouverte par Roland Barthes (avec les fonctions, catalyses et cardinales, d'une part, les indices, proprement dits et informants, d'autre part) pourrait se révéler fructueuse.

Dans le second cas, une action est lisible : Chauvin vient rejoindre Anne, rétablissant ainsi un contact suspendu depuis un long moment. Le récit progresse donc; de l'information est produite. Néanmoins une durée sensible se manifeste encore, due cette fois-ci, me semble-t-il, à la profondeur de champ d'une part, à la vacuité du décor de cette maison abandonnée d'autre part. Le spectateur a tout loisir de se concentrer sur le déroulement continu de l'action, de suivre Chauvin depuis l'embrasure de la porte jusqu'au moment où il rejoint Anne. On pourrait ici faire appel à la loi d'encombrement de l'image, proposée par Claudine de France, et avancer une seconde règle : pour un temps chronométrique donné, l'effet de durée est inversement proportionnel à l'encombrement de l'image. Ce principe s'articule, bien entendu, directement sur le taux d'information, car une image encombrée est une image riche d'informations dont la saisie demande du temps. L'image dépouillée, frappée de vacuité, s'offre plus immédiatement à la lecture.

Le film de Peter Brook joue pleinement sur cette vacuité, soulignée par la profondeur de champ. S'il s'agit là, probablement, d'un parti-pris esthétique, il travaille en même temps à produire un effet de durée et, au-delà, de brouillage temporel.

Dès lors, le film produit structurellement, et au niveau du seul récit premier, cette ambivalence paradoxale faite d'un temps irréversible, inéluctable et tragique confronté à un temps qui se dilate en une continuelle expansion, visant à absorber le premier, et qui pourtant sera rejoint par lui.

En ce sens, il est tout à fait révélateur que le film s'achève par là où il a commencé : sur un cri, cette fois-ci poussé par Anne Desbaresdes en écho à celui de l'inconnue. Ce bouclage des deux temporalités, en ce qu'elles se rejoignent dans ce cri, boucle aussi le film.

De l'examen de cette adaptation, quels enseignements peut-on tirer? Il me semble qu'ils sont de deux ordres. Le premier est d'ordre méthodologique. Le passage de l'écrit à l'écran me paraît 
devoir être pensé non pas en termes de comparaison ou d'équivalence, mais en termes d'opérations. Cela suppose que l'on conçoit le roman non comme un modèle mais comme un vaste réservoir d'instructions. Le film choisit de faire siennes certaines d'entre elles et les traite en prenant en charge sa propre spécificité. Ici c'est la question de la temporalité, et plus particulièrement de la durée, qui est travaillée dans cette perspective.

De là découle un second enseignement, d'ordre théorique celui-ci. Le travail spécifique du film oblige à situer la réflexion au plus près de la singularité du médium. L'approche de la durée au cinéma demande donc à être posée sur la base d'une double articulation, celle propre au plan, celle propre au syntagme. Par là, elle prend en compte l'expérience phénoménologique du spectateur, inscrit dans l'«ici-maintenant» de la perception, et permet de faire apparaître une durée en prise sur l'acte de monstration (celle interne au plan) ainsi qu'une autre davantage liée à la narration (celle du syntagme).

En ce sens, Moderato cantabile, le film aussi bien que le roman, comme son titre l'indique, serait donc bien un film sur le tempo.

Université Lumière - Lyon II

\section{NOTE}

1 Selon l'appellation de Jean-Paul Simon : «Remarques sur la temporalité cinématographique dans les films diégétique», in Cinémas de la modernité : films théories (colloque de Cerisy dirigé par Dominique Château, André Gardies, François Jost), Paris : Klincksieck, 1981.

\section{OUVRAGES CITÉS}

Barthes, Roland. «Introduction à l'analyse structurale des récits», in Poétique du récit. Paris : Seuil, 1977.

Duras, Marguerite. Moderato Cantabile. Paris : Minuit, 1990.

Genette, Gérard. Figures III. Paris : Seuil, 1972. 